\title{
IMPROVED ELECTION OF CLUSTER HEADS IN LEACH
}

\author{
Chaima Bejaoui \\ Sfax University \\ National School of Engineers of Sfax \\ Road Soukra km 4 - 3038 Sfax, Tunisia \\ Email: bejaouichaima@gmail.com
}

\author{
Alexandre Guitton \\ Clermont University LIMOS CNRS \\ Cezeaux Scientific Complex \\ 63177 Aubiere cedex, France \\ Email: alexandre.guitton@univ-bpclermont.fr
}

\author{
Abdennaceur Kachouri \\ Gabes University \\ Higher Institute of Industrial Systems \\ 6032 Gabes, Tunisia \\ Email: abdennaceur.kachouri@enis.rnu.tn
}

\begin{abstract}
Wireless Sensor Networks (WSNs) are a promising technology to monitor distant or inaccessible areas. As nodes have a limited energy supply, many routing protocols are based on a clustering mechanism: some nodes are elected as cluster heads and have to deal with most of the communication burden of the network, while the other nodes perform only simple operations. In this paper, we propose a new election mechanism with important features: it ensures that all nodes are in range of a cluster head while keeping the number of cluster heads low, it takes into account the residual energy of nodes, and it requires a small communication overhead. We compare the performance of our election mechanism with an optimal election, as well as with the election mechanism of LEACH, which is the main clustering algorithm for WSNs.
\end{abstract}

\section{INTRODUCTION}

Wireless Sensor Networks (WSNs) are one of the most promising technology to monitor distant or inaccessible areas [1]. They are used in several application domains, including military surveillance, precision agriculture and healthcare monitoring. Nodes in WSNs are characterized by their sensing, computation and communication capabalities. Nodes are usually low cost, easy to deploy, and are equipped with batteries which provide them with a limited autonomy.

Nodes spend a significant part of their energy on wireless communications. To reduce the energy consumption of communications, many wireless protocols use a hierarchical approach, where nodes can be either members or cluster heads. Members have a limited burden: they simply forward their data to their cluster heads, and go to sleep in order to save energy. Cluster heads receive the data from their members, aggregate them, and transmit them to a special node called the sink. Cluster heads consume more energy than members, but nodes change their roles often to balance their energy consumption.

In this paper, we focus on the election of cluster heads. We propose a new election mechanism that has three important features: (i) it ensures that all members are in range of a cluster head, (ii) it aims to balance the energy consumption of cluster heads, and (iii) it requires a small communication overhead. We compare our new election mechanism with the election mechanism of LEACH [2], which is the main clustering algorithm for WSNs.

This paper is organized as follows. Section 2 describes the related work on clustering protocols and on protocols based on LEACH. Section 3 describes the drawbacks of most LEACHbased clustering protocols. Then, we propose an optimal election algorithm (based on integer linear programming), as well as our protocol. Section 4 presents our simulation results, and compares the election mechanism of our protocol with the election mechanism of LEACH. Finally, Section 5 concludes our work.

\section{RELATED WORK}

In this section, we describe several clustering protocols for WSNs. We classify these protocols into two categories: LEACH-based protocols, and other related protocols.

\section{A. LEACH-based clustering protocols}

LEACH [2] [3] was the first clustering protocol for WSNs. Time in LEACH is divided into rounds, and each round is divided into an election phase and a communication phase. During the election phase, each node $n$ starts by computing a threshold $T(n) . T(n)$ is equal to $k /(N-k(\operatorname{rmod} N / k))$, where $\mathrm{N}$ is the number of nodes in the WSN, $r$ is the round number and $k$ is a predefined constant indicating the desired percentage of cluster heads. Then, each node decides to become a cluster head with probability $T(n)$. When a node is a cluster head, it broadcasts an ADV message and nodes that are not cluster heads reply with a JOIN message to one of the cluster heads in range. Finally, the cluster head broadcasts a schedule to all its members, where each member is given a dedicated time slot for the communication phase. During the communication phase, members are allowed to communicate only during the dedicated time slot. Moreover, members can only communicate with their cluster heads. Outside of their dedicated time slots, members save energy by deactivating their radio module. At the end of the communication phase, the cluster head communicates with the sink, which is supposed to be accessible directly through a long-distance high-power communication.

Many researchers have aimed to improve LEACH by modifying its election mechanism, its routing mechanism, or both. As we focus in this paper on the election mechanism, we only describe here the protocols that propose significant improvements on the election mechanism of LEACH.

ALEACH [4] adds the fraction of the remaining energy of node $n$ into the computation of $T(n)$. In this way, a node that has little remaining energy has a reduced probability of becoming a cluster head. 
EL-LEACH [5] makes several modifications in LEACH. First, it integrates the fraction of the remaining energy of node $n$ (similarly to ALEACH)into $T(n)$. Second, it introduces a mechanism where all cluster heads in range compete: each ADV message contains a metric that includes both the fraction of remaining energy of the cluster head, and its distance to the sink. Only the nodes having the largest metric remain cluster heads for this round. Third, the members select the cluster head having the largest metric.

LEACH-P [6] also uses neighborhood information in order to reduce the number of cluster heads. Each node $n$ computes $T(n)$ according to its residual energy and number of neighbors, and broadcasts this value. The nodes having the largest value $T(n)$ in their neighborhood become cluster heads.

ACHTH-LEACH [7] uses geographic information to optimize the cluster head placement. Nodes that are close to the sink choose the sink as cluster head, while nodes that are far away from the sink use a centralized algorithm for the election. The algorithm for the election of the first round combines $\mathrm{K}$ means with a greedy approach. The algorithm for the other rounds is a weighted round-robin based on residual energy.

\section{B. Other related protocols}

PEGASIS [8] computes a chain among nodes in order to reach the sink: each node is allowed to receive and to transmit only to its immediate predecessors and successors on the chain, and saves energy the remainder of the time.

TEEN [9] is a reactive protocol that builds clusters using two thresholds: hard and soft. The hard threshold ensures that the number of transmitted data is above a threshold, so that there is no cluster head with few members. The soft threshold allows the data generation interval to change dynamically. As in LEACH, cluster heads gather data from all their members and send them to the sink.

HEED [10] proposes an election mechanism based on several iterations. At each iteration, nodes compute a probability to become a cluster head which depends on their residual energy, on their degree and on the distance from their neighbors to the sink. When a node does not have a cluster head in range at an iteration, another iteration is performed where the probability of each node to become a cluster head is doubled.

DEEC [11] and SEP [12] assume that nodes are divided into two categories: normal nodes having limited energy, and advanced nodes having more energy than normal nodes. The election takes into consideration the type of nodes and the residual energy of nodes with respect to the average energy of the network.

\section{PROPOSITION}

Most LEACH-based clustering protocols in the litterature modify the election mechanism of LEACH in three ways: (i) they add more steps in the election mechanism so that nodes can collaborate with their neighbors to form cluster heads, (ii) they modify the computation of the threshold $T(n)$, or (iii) they are centralized and have the knowledge of the topology. These modifications have several drawbacks.
- Protocols that add more steps to the election mechanism introduce more control overhead and complexity to LEACH. Protocols that do not add steps in the election mechanism might produce isolated nodes, that is members that are not in range of a cluster head.

- Protocols that modify the computation of threshold $T(n)$ generally include the residual energy or node density. However, it is still possible for nodes having the lowest energy to be elected as cluster heads.

- Protocols that are centralized are less robust than the initial LEACH, which is distributed. Moreover, the knowledge of the topology requires several control messages.

In the following, we propose two modifications of the election mechanism of LEACH: an optimal election and our protocol.

- The optimal election uses an integer linear program to compute a trade-off between number of cluster heads and residual energy of the cluster heads. It is centralized and requires the knowledge of the whole topology. We use this optimal election as a comparison basis for LEACH and our protocol.

- $\quad$ Our protocol modifies the election of LEACH by allowing nodes to refrain from sending ADV messages, if they detect several cluster heads in the neighborhood, thus reducing the number of cluster heads of LEACH. Moreover, it allows isolated nodes to become cluster heads in order to be allowed to communicate with the sink.

\section{A. Optimization by ILP}

In order to compute the optimal number of cluster heads, we decided to use an integer linear program that takes as input the whole network topology and the residual energy of each node. This linear program computes a trade-off between the number of cluster heads and their energy. Objective function and the constraints of our ILP program are summarized below.

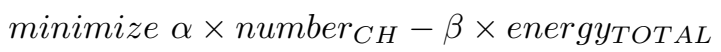

$$
\begin{gathered}
\forall n \in N, \sum_{n \in N}(1-f(n, v)) \geq 1 \\
\forall n, v \in N^{2}, f(n, v) \geq 1-\operatorname{neigh}(n, v) \\
\forall n, v \in N^{2}, f(n, v) \geq 1-C H(v) \\
\forall n, v \in N^{2}, f(n, v) \leq 2-\operatorname{neigh}(n, v)-C H(v) \\
\text { number }_{C H}=\sum_{n \in N} C H(n) \\
\forall n \in N, \operatorname{energy}_{C H}(n) \leq \operatorname{energy}(n) \\
\forall n \in N, \text { energy }_{C H}(n) \leq \text { energy } \\
\forall n \in N \text { M } \\
\forall n \text { energy } \\
\forall n(n)
\end{gathered}
$$

The inputs of this program are as follows; $N$ is the set of nodes, energy $(n)$ is the current energy of a node and 
energy $y_{M X}$ is the maximal energy of a node in the current round. The variables that we use are cited below. number ${ }_{C H}$ defines the number of CHs. energy $y_{T O T A L}$ corresponds to the total energy of all CHs. energy $y_{C H}(n)$ defines the energy of $n$ if it is a $\mathrm{CH}$ and 0 otherwise. Finally, $f(n, v)$ is a binary variable equal to zero if $n$ is $\mathrm{CH}$ and $v$ is neighbor of $n$.

In the objective function of our ILP program, we weight the number of $\mathrm{CHs}$ by $\alpha$ and the total energy by $\beta$. The constraints (1), (2), (3) and (4) model the fact that each node is either a $\mathrm{CH}$ or it is a neighbor of a $\mathrm{CH}$. The fifth constraint calculates the total number of CHs. The sixth and seventh constraints ensure that if a node is not a $\mathrm{CH}$, then its energy equals 0 , and if it is a $\mathrm{CH}$, energy $y_{H}(n)$ is equal to the energy of $n$. The last constraint calculates the total energy of $\mathrm{CHs}$ in the system.

\section{B. Our protocol}

Our protocol modifies two parts of the election mechanism of LEACH: the decision to become cluster head, and the join process.

In our protocol, the decision to become a cluster head is still made at the beginning of a round, according to the threshold $T(n)$, but the ADV message is not sent immediately: each node delays the ADV transmission by a random delay. When a node receives more than th ADV messages before sending its own ADV, th being a parameter of our protocol, the node decides that there are too many cluster heads in the neighborhood, and decides to become a member instead of a cluster head. Thus, this node decides not to send its own ADV anymore.

In our protocol, the join process allows isolated nodes (that is, nodes that did not receive ADV messages) to become cluster head, and to start gathering their own members. This decision is made towards the end of the election, when the ADV messages from the initial cluster heads are expected to be received.

Notice that our distributed protocol does not introduce control messages (as it uses overhearing of ADV messages) and is supposed to greatly reduce the number of isolated nodes. It is still possible for a node to be isolated if a JOIN message is not received by a cluster head, but this situation is not expected to occur frequently. It is also possible that the total number of cluster heads obtained is increased with respect to LEACH, as our solution to the problem of isolated nodes increases the number of cluster heads. However, we believe that it is more important to allow every node of the network to communicate at every round, rather than to have a minimum number of cluster heads.

\section{Simulation Results}

In this section we present simulation results together with analyzes. We use the NS2 simulator to simulate our proposition and LEACH protocol. Nodes are deployed in an area of 600 $\mathrm{m} \times 600 \mathrm{~m}$, and the network consists of 50, 75 and 100 nodes scattered uniformly at random in the area. Each result is averaged ones 100 repetitions, and each repetitions consist of 1000 seconds. Each node is equipped with initial energy of 2 Joules. We use the MAC layer of 802.11.

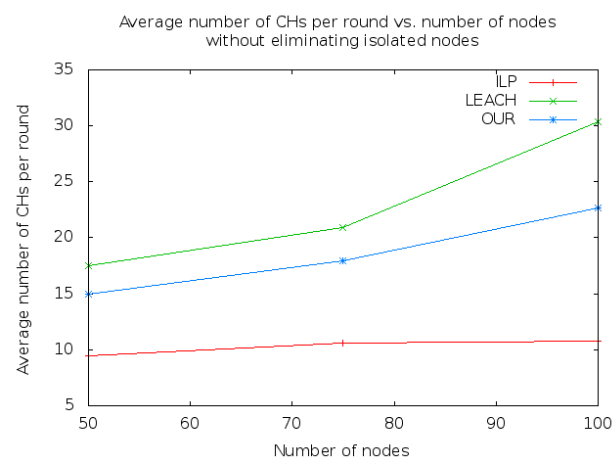

Fig. 1. Average number of $\mathrm{CHs}$ per round vs. number of nodes without isolated nodes.

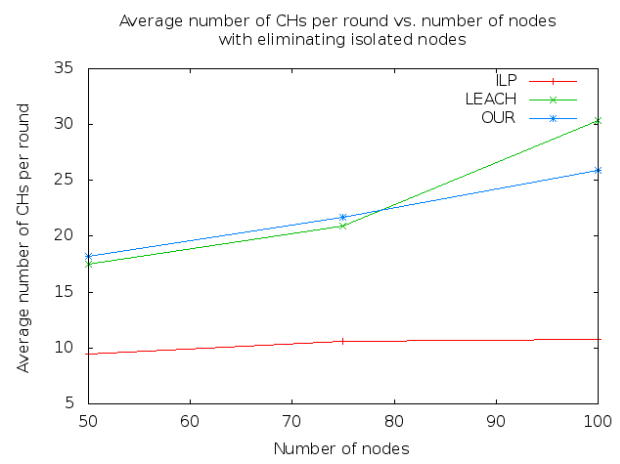

Fig. 2. Average number of $\mathrm{CHs}$ per round vs. number of nodes with isolated nodes.

We evaluate two parameters in our simulation such as average number of $\mathrm{CHs}$ per round and average number of isolated nodes.

Figure 1 shows the average number of $\mathrm{CHs}$ per round as a function of the number of nodes for the LEACH protocol (without eliminating the isolated nodes), our proposal and the ILP program. We should also note that the number of $\mathrm{CHs}$ in our proposition is less than with $\mathrm{LEACH}$, because of the emission of ADV at different times, which allows nodes to wait for the reception of other ADVs to take the decision to be a $\mathrm{CH}$ or not. We show also that ILP is less than the two protocol because it represents the optimal result. We also note that for 100 nodes, our algorithm reduces the number of $\mathrm{CHs}$ of up to $10 \%$ compared to LEACH.

Figure 2 shows the average number of $\mathrm{CHs}$ per round as a function of the number of nodes without eliminating the isolated nodes, for the three protocols. We can see that the number of $\mathrm{CHs}$ in our algorithm is slightly higher than the number of $\mathrm{CHs}$ in $\mathrm{LEACH}$, because of the elimination of isolated nodes. Some of the isolated nodes become $\mathrm{CHs}$ in the JOIN period and some become members of the new $\mathrm{CHs}$, which automatically increases the number of $\mathrm{CHs}$. Against by this increase is not noticed at simulation of 100 nodes because the $\mathrm{LEACH}$ protocol requires a much larger number of $\mathrm{CHs}$ with this number of nodes.

Figure 3 represents the average number of isolated per round according to the number of $\mathrm{CHs}$ for $\mathrm{LEACH}$. Note that the ILP cannot yield isolated nodes, and our protocol did not 


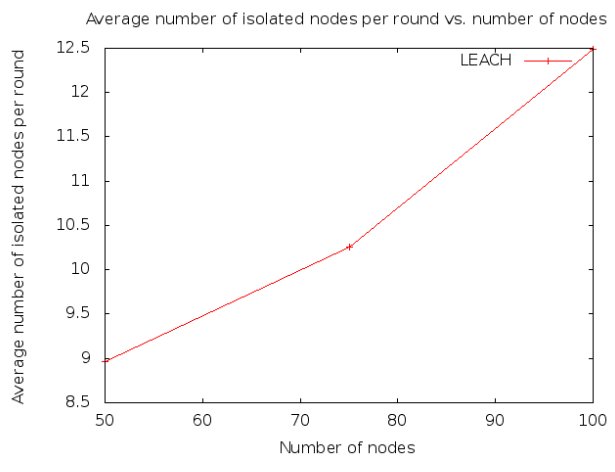

Fig. 3. Average number of isolated nodes per round vs. number of nodes.

yield isolated nodes during the simulations, so they do not appear in the figure. However, the average number of isolated nodes of LEACH varies between 8 and 12 which make our proposition more usable than LEACH.

\section{Conclusions}

In this paper, we discussed clustering protocols based on LEACH. We proposed an integer linear program to compute the optimal set of clusters, and we designed a protocol that reduces the number of $\mathrm{CHs}$ without producing isolated nodes. Simulation results showed that our protocol reduces the number of CHs (which reaches $10 \%$ of reduction for 100 nodes) compared with LEACH. Our protocol presents an optimal result (number of isolated nodes is null) for isolated nodes with a small increase of number of the $\mathrm{CHs}$ for the simulations of 50 and 75 nodes. The perspective of this work includes the improvement of the election phase to be adapted to wireless multimedia sensor networks.

\section{REFERENCES}

[1] I.F Akyildiz, W. Su, Y. Sankarasubramaniam and E. Cayirci, Wireless sensor networks: a survey, Comput. Networks, ELSEVIER, 2002.

[2] W. Heinzelman, Energy-efficient communication protocol for wireless sensor networks, PhD. Thesis, 2000.

[3] W. Heinzelman, A. Chandrakasan and H. Balakirshnan, ApplicationSpecific protocol architectures for wireless networks, Hawaii International Conference System Sciences, 2000.

[4] Md. Solaiman, T. Dey and R. Biswas, ALEACH: Advanced LEACH Routing Protocol for Wireless Microsensor Networks, International Conference on Electrical and Computer Engineering (ICECE), 2008.

[5] T. Ngo Quynh, K-H. Phung and H. Vu Quoc, Improvement of Energy Consumption and Load Balance for LEACH in Wireless Sensors Networks, ICT Convergence (ICTC), IEEE, 2012.

[6] W. Wang and Y. Peng, LEACH Algorithm Based on Load Balancing, TELKOMNIKA, 2013.

[7] L-Q. Guo, Y. Xie, C-H. Yang and Z-W. Jing, Improvement on leach by combining adaptive cluster head ele ction and two-hop transmission, the Ninth International Conference on Machine Learning and Cybernetics, 2010.

[8] S. Lindsey and C. Raghavendra, PEGASIS: Power- Efficient Gathering in Sensor Information Systems, Aerospace Conference Proceedings,IEEE, 2002.

[9] A. Manjeshwar and D. Agrawal, TEEN: A protocol for enhanced efficiency in wireless sensor networks, Proceeding of the 1 th International Workshop on Parallel and Distributed Computing Issues in Wireless Networks and Mobile Computing01, 2001.
[10] O. Younis and S. Fahmy, Distributed Clustering in Ad-hoc Sensor Networks: A Hybrid, Energy-Efficient Approach, Proceedings of IEEE INFOCOM, 2004.

[11] Q. Li, Z. Qingxin and W. Mingwen, Design of a distributed energy efficient clustering algorithm for heterogeneous wireless sensor networks, Computer Communications, 2006.

[12] G. Smaragdakis, I. Matta and A. Bestavros, SEP: A Stable Election Protocol for clustered heterogeneous wireless sensor networks, Second International Workshop on Sensor and Actor Network Protocols and Applications (SANPA), 2004. 